\title{
Néolithisation et néolithique de Tunisie : Les sites et leur faune domestique
}

Yosra Dridi et Nabiha Aouadi

\section{OpenEdition}

1 Journals

Édition électronique

URL : https://journals.openedition.org/encyclopedieberbere/2722

DOI : 10.4000/encyclopedieberbere.2722

ISSN : 2262-7197

Éditeur

Peeters Publishers

\section{Édition imprimée}

Date de publication : 5 octobre 2012

Pagination : $5481-5488$

ISBN : 978-90-429-2718-6

ISSN : 1015-7344

Référence électronique

Yosra Dridi et Nabiha Aouadi, « Néolithisation et néolithique de Tunisie : Les sites et leur faune domestique », Encyclopédie berbère [En ligne], 34 | 2012, document N44, mis en ligne le 15 décembre 2020, consulté le 17 février 2022. URL : http://journals.openedition.org/encyclopedieberbere/2722 ; DOI : https://doi.org/10.4000/encyclopedieberbere.2722

Ce document a été généré automatiquement le 17 février 2022.

(C) Tous droits réservés 


\title{
Néolithisation et néolithique de Tunisie : Les sites et leur faune domestique
}

\author{
Yosra Dridi et Nabiha Aouadi
}

\section{Introduction}

1 En Tunisie plusieurs sites de l'Holocène moyen et final ont été anciennement attribués au Néolithique. Ces attributions culturelles reposaient sur la présence d'outils lithiques tels que les pointes de flèche, les haches et herminettes polies, le matériel de broyage, certains microlithes géométriques (rectangles) ainsi que sur des tessons de céramiques. Concernant, la définition même d'un site néolithique, nous optons désormais pour la présence certaine d'un mode de vie fondé partiellement ou intégralement sur la production de substances animale et végétale. Les sociétés dites néolithiques sont celles où l'élevage d'animaux domestiques est bien assuré. Ainsi, donc, nous ne décrivons ici que les sites ayant livré de la faune domestique en Tunisie, en nous appuyant sur la révision des témoins archéozoologiques disponibles (Dridi 2011).

\section{Redeyef (Table Sud)}

2 Ayant la forme d'un puissant abri sous roche, le site de Redeyef est situé à environ $1 \mathrm{~km}$ au nord-ouest de la ville minière de Redeyef dans le sud-ouest tunisien. E.-G. Gobert (1912) le découvrit et y pratiqua des sondages. Il y identifia deux niveaux archéologiques superposés. Le premier appartenant au Capsien typique, le second, plus récent appartenant au Néolithique (Fig. 2.). Plus tard, dans les années 1931-1932, R. Vaufrey y réalisa de nouveaux sondages. Ce matériel contribuera, en association avec d'autres documents de sites épipaléolithiques dans la région, à la définition du Néolithique de tradition capsienne (Vaufrey 1933, 1955). L'industrie lithique du niveau Capsien aurait les mêmes affinités que celles de Bortal Fakher d'après N. Rahmani (2003). Le niveau rattaché au Néolithique a été considéré comme un Néolithique ancien 
par G. Camps (1974). Des restes humains avaient été également exhumés du site par E.G. Gobert (1912). Certains déposés à l'Institut de Paléontologie Humaine ont fait l'objet d'un examen par J.-L. Heim (1979). Il s'agit de populations négroïdes. Les rares tessons de céramique issus du niveau néolithique ont été étudiés par Aumassip (1969-1970) et montrent une poterie "fortement élaborée ». La faune sauvage présente un caractère plus archaïque que celle examinée pour les deux sites suivants. En effet, elle renferme Bos primigenius, Bos sp., Alcelaphus buselaphus, Ammotragus lervia, Equus sp., Ceratotherium mauritanicum, Canis aureus, Vulpes sp., Felis caracal, Herpestes ichneumon, plusieurs espèces de rongeurs et des hélicidés terrestres (Tableau 1). Mais l'intérêt des nouvelles recherches est d'avoir mis en évidence quelques pièces anatomiques se rapportant à une faune domestique comprenant uniquement Ovis sp. C'est la première fois que cela est souligné en Tunisie.

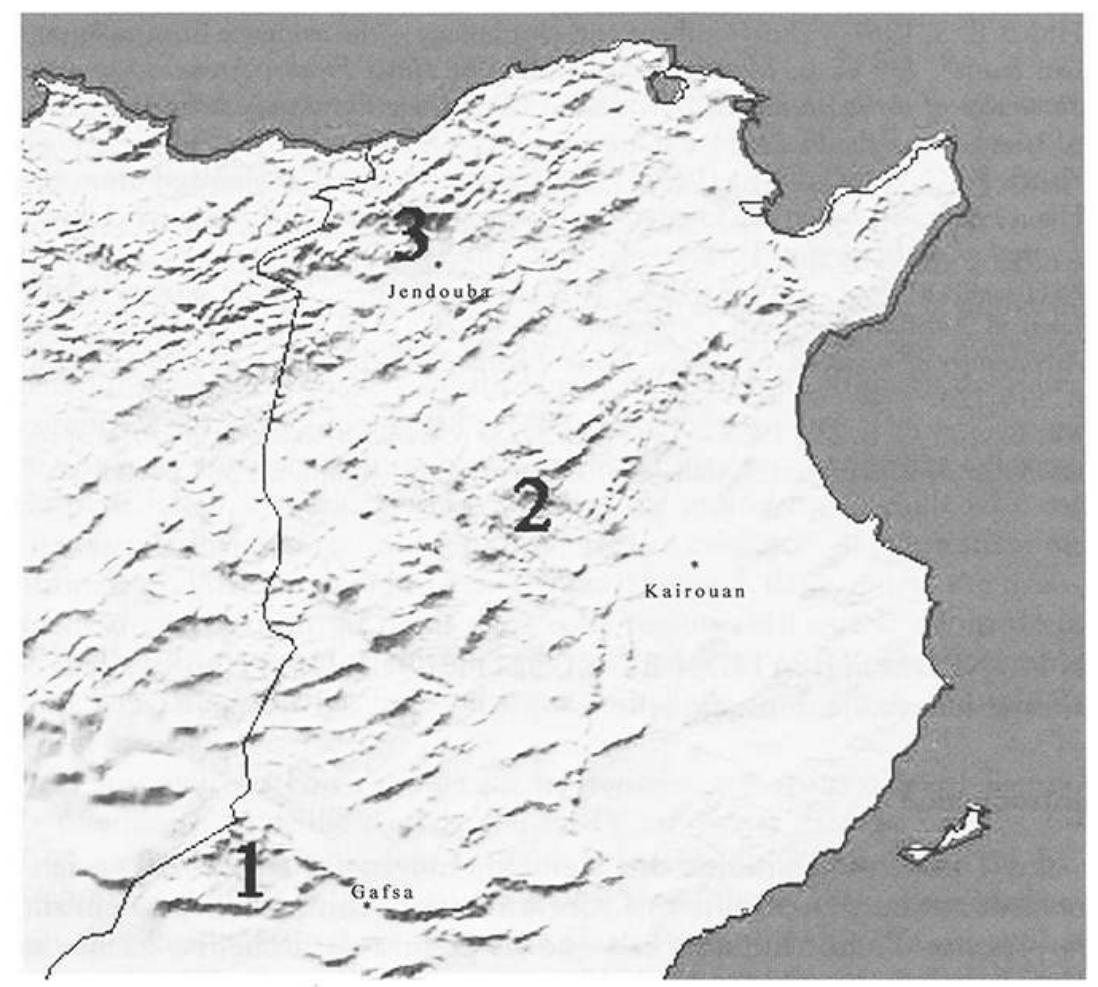

Fig. 1. SITES NÉOLITHIQUES DE TUNISIE. 


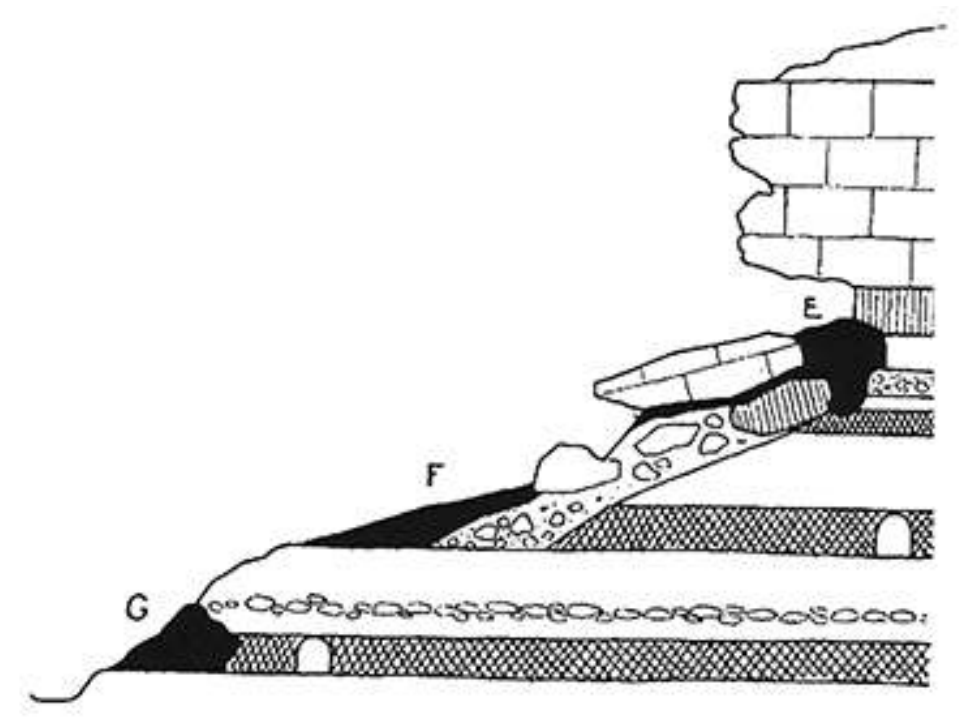

FIg. 2 : COUPE DE L'ABRI DE REDEYEF AVEC SES DEUX NIVEAUX : G ET F : CAPSIEN TYPIQUE, E : NÉOLITHIQUE (D'APRÈS GOBERT, 1912).

\section{Kef el-Agab*}

Le site connu du Kef el-Agab est formé d'une vaste grotte creusée dans les dolomies rouges oxydées et diaclasées du Jebel Kef el-Agab, il se prolonge à l'extérieur par une rammadiya couvrant le talus. Il est situé à environ $4 \mathrm{~km}$ au nord-ouest de la ville de Jendouba, sur la rive gauche d'un ravin et s'ouvrant vers le sud-ouest. Il a été découvert et fouillé par P. Bardin au cours des années 1947-1948. Les fouilles de P. Bardin ont abouti au retrait presque total du contenu de la grotte et de son vestibule et au creusement d'une énorme tranchée dans le talus. P. Bardin publia une première note en 1951 puis, dans une publication plus détaillée datée de 1953, il donna tous les résultats de ses recherches et la manière dont il a procédé lors de ses fouilles (Zoughlami et al. 1989). Le site a livré une importante collection faunique, lithique, et quelques tessons de céramique. D'après les fouilles de Bardin, des restes humains pêlemêle auraient été retrouvés dans quatre sinus des parois du vestibule et dans les dépôts archéologiques du talus, à l'entrée de la grotte. L'étude de ces individus a été entreprise plusieurs fois. H.-V. Vallois (1953) les attribue au type dit "Méditerranéen primitif». M.-C. Chamla (1978) note la présence de deux types humains : un type dit " Méchtoïde » et un autre dit « Proto-méditerranéen ». D'autre part, les documents osseux et lithiques ont été recueillis sans aucune indication stratigraphique, ni même de provenance approximative. Récemment, de nouveaux sondages ont été pratiqués dans le talus (Aouadi 2010). Plusieurs niveaux d'occupations ont été identifiés. L'industrie lithique est sur silex, calcaire et sur fragments de coquille d'Unio, ce qui constitue une grande nouveauté. La sépulture d'un nouvel individu dont le squelette avait été conservé en connexion anatomique a été retrouvée dans le sondage $\mathrm{S} 1$ en position fléchie latérale (Fig. 3.). Parmi les 73 documents fauniques déterminés (NRD) issus de ces sondages signalons 25 restes crâniens et 61 restes post-céphaliques. Les restes crâniens identifiés, appartiennent au groupe Capra/Ovis (20\%) alors que $20,5 \%$ seulement des restes postcrâniens sont ceux d'ovi-caprinés domestiques (Fig. 4.). Nous notons que la malacologie est composée dans les niveaux supérieurs d'une majorité de coquilles d'Unio (mollusque d'eau douce qui vivait et continue de vivre dans la vallée de la Medjerda), puis vers les 
niveaux inférieurs, d'espèces d'Helix terrestres telles que Leucochroa candidissima, Helix aspersa et Eobania vermiculata. Les fouilles de P. Bardin ont donné un total de 277 pièces fauniques déterminables. Le groupe Capra/Ovis forme $14,8 \%$ des restes crâniens déterminables et $3 \%$ des restes post-crâniens déterminables. Les restes d'animaux sauvages sont dominants et sont représentés par les Bovini, Alcelaphini, Reduncini, Antilopini, Caprini, Suidés, Canidés, Félidés, Hyénidés, Herpestidés, Lagomorphes, Insectivores, Rongeurs et l'autruche.

Tableau 1 : Liste faunique de trois sites néolithiques tunisiens.

\begin{tabular}{|c|c|c|c|c|c|}
\hline $\begin{array}{l}\text { Groupe } \\
\text { animal }\end{array}$ & Tribu & Genre et Espèce & 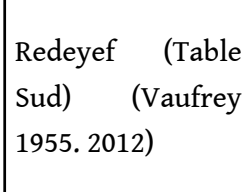 & $\mid \begin{array}{ll}\text { Kef } & \text { el- } \\
\text { Agab } & \\
\text { (Dridi } & \\
2012) & \end{array}$ & $\begin{array}{l}\text { Doukanet } \quad \text { el- } \\
\text { Khoutifa } \\
\text { 2012) }\end{array}$ \\
\hline \multirow[t]{13}{*}{ Bovidé } & & Bos primigenius & * & * & * \\
\hline & Bovini & Bos sp. & * & & $*$ \\
\hline & & Syncerus caffer & & * & \\
\hline & Alcelaphini & Alcelapbus buselaphus & * & * & * \\
\hline & & Damaliscus sp. & & * & * \\
\hline & Reduncini & Redunca redunca & & $*$ & $*$ \\
\hline & & Kobus kob & & & $*$ \\
\hline & & Gazella dorcas & & * & \\
\hline & Antilopini & Gazella cuvieri & & * & \\
\hline & & Gazella sp. & & & $*$ \\
\hline & & Ammotragus lervia & * & * & $*$ \\
\hline & Caprini & ovis sp. & * & * & $*$ \\
\hline & & Capra sp. & & * & * \\
\hline Suidé & & Sus scrofa & & * & \\
\hline \multirow[t]{2}{*}{ Equidé } & & Equus asinus & & & * \\
\hline & & Equus sp. & * & & * \\
\hline Rhinocérotidé & & $\begin{array}{l}\text { Ceratotherium } \\
\text { mauritameum }\end{array}$ & * & & \\
\hline Canidés & & Canis aureus & * & * & \\
\hline
\end{tabular}




\begin{tabular}{|c|c|c|c|c|}
\hline & Canis familiaris & & & * \\
\hline & Vulpes sp. & * & & \\
\hline Hyénidé & Crocuta crocuta & & $*$ & \\
\hline \multirow[t]{2}{*}{ Félidé } & Felis caracal & $*$ & & \\
\hline & Felis sp. & & $*$ & \\
\hline Herpestidé & Herpestes ichneumon & * & * & \\
\hline \multirow[t]{2}{*}{ Lagomorphe } & Lepus sp. & & $*$ & \\
\hline & Oryctolagus cuniculus & & & * \\
\hline Insectivore & Atelerix algirus & & $*$ & * \\
\hline \multirow[t]{4}{*}{ Rongeur } & Hystrix cristata & $*$ & $*$ & \\
\hline & Ctenodactylus gututi & $*$ & & \\
\hline & Meriones shawi & $*$ & & \\
\hline & Jaculus jaculus & $*$ & & \\
\hline Reptile & Testudo graeca & & & $*$ \\
\hline Oiseau & Struthio $s p$. & & * & * \\
\hline \multirow[t]{5}{*}{ Hélicidé } & $\begin{array}{l}\text { Leucochroa } \\
\text { candidissima }\end{array}$ & $*$ & $*$ & * \\
\hline & Helix melanojtoma & $*$ & & * \\
\hline & Helix aspersa & & $*$ & * \\
\hline & Eobania vermiculata & $*$ & $*$ & * \\
\hline & Rumina decollata & & $*$ & \\
\hline Moule & Unio sp. & & $*$ & \\
\hline
\end{tabular}




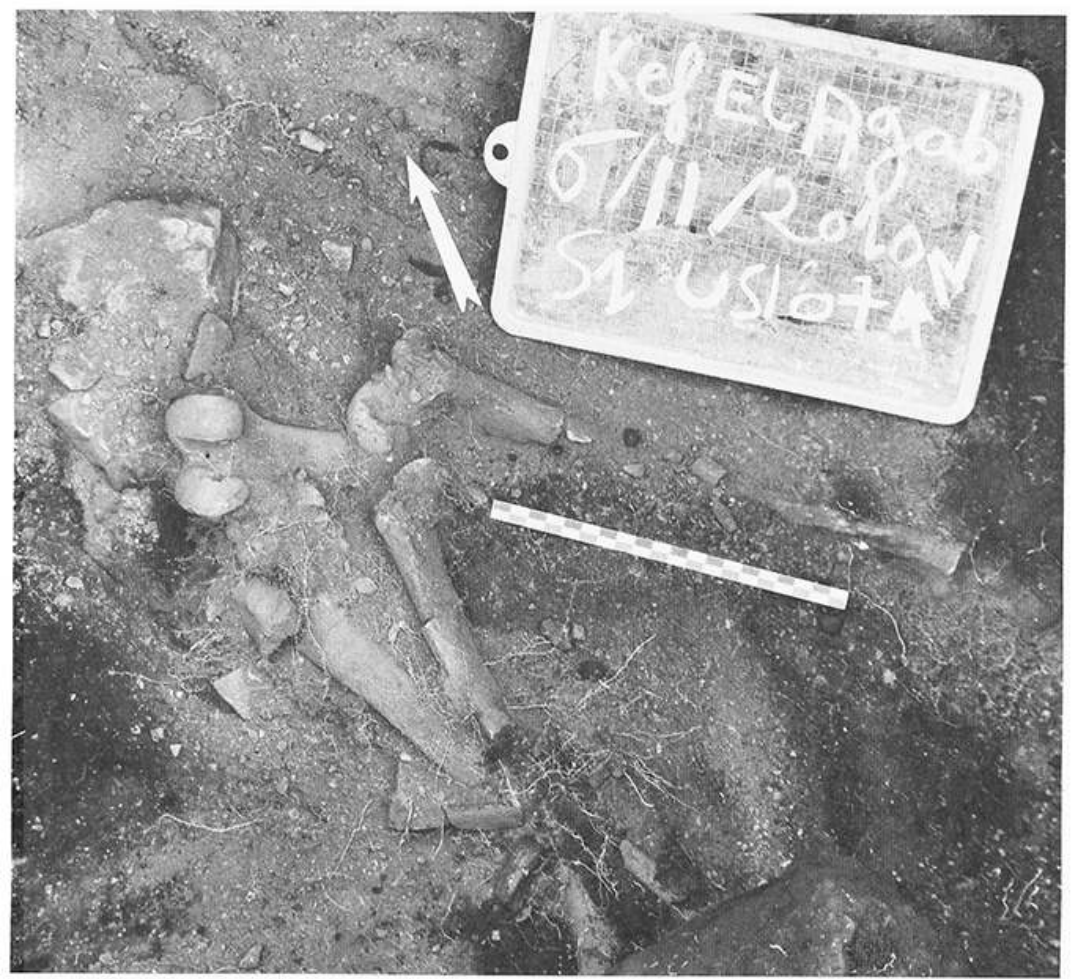

Fig. 3. Sépulture humaine trouvée dans le sondage s1 de kef el-agab (Photo aouadi 2010).

\section{Doukanet el-Khoutifa}

Dans la région de Siliana, le site de Doukanet el-Khoutifa est situé dans le massif de Kef el-Guéria, faisant partie de la dorsale tunisienne (Zoughlami et al. 1998). Il occupe une plate-forme de quelque $2000 \mathrm{~m}^{2}$; il a été découvert et fouillé au début des années 1970 par J. Zoughlami $(1978,2009)$. Il s'agit d'une rammadiya, constituée en grande partie de coquilles d'hélix et d'ossements animaux, auxquels se mêlent en grandes quantités de pierres brûlées, des outils lithiques, osseux et des tessons de céramique à fond conique. Plusieurs sépultures humaines ont été également découvertes sous un rocher mais les ossements n'étaient pas tous en connexion anatomique (Roudesli-Chebbi \& Zoughlami 2004 ; Zoughlami 2009). Ces individus sont des Proto-méditerranéens. Deux unités stratigraphiques distinctes se superposent. La couche de couleur jaune la plus ancienne a été datée sur charbons entre $6750 \pm 200$ BP soit $6021-5317$ cal BC (2 sigma). La couche noire suivante, la plus importante, d'épaisseur $1,6 \mathrm{~m}$, plus récente, a été datée sur charbons entre $6100 \pm 100$ BP soit $5297-4792$ cal BC (2 sigma) et $6150 \pm 100$ BP soit 5317-4838 cal BC (2 sigma). La faune sauvage est formée de Bos primigenius, un bovini de petite taille, Alcelaphus buselaphus, Damaliscus sp., Kobus kob, Redunca redunca, Gazella sp., Ammotragus lervia, Equus asinus, Equus sp., Oryctolagus cuniculus, Struthio sp. Testudo graeca. La faune domestique comporte Canis familiaris, Ovis sp., Capra sp. (Fig. 5.). 


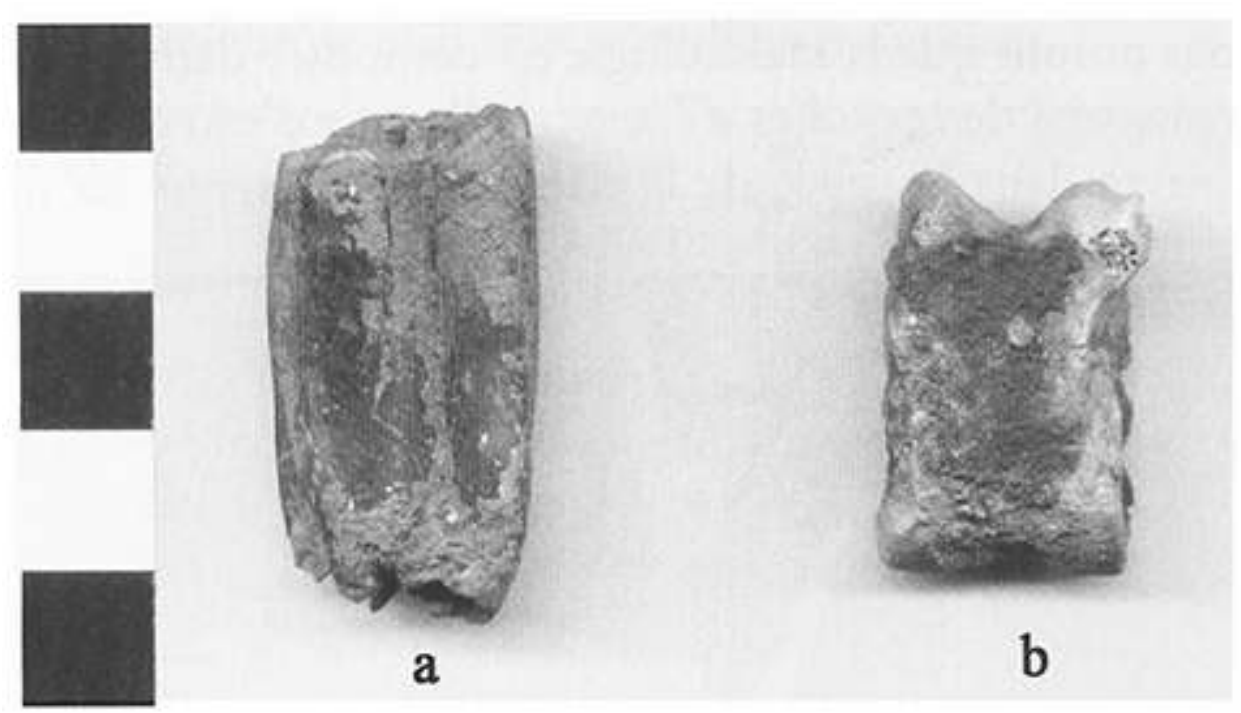

Fig. 4. PHOto de RESTES faUniques d'oVi-CAPRINÉS domestiques de KEF EL-AgAB (AOUAdi 2010). A : $M 3$ SUPÉRIEURE (S1 BORD EXTERNE, US1, $\mathbf{N}^{\circ} 42$ ).

$B$ : TALUS $\left(\mathbf{N}^{\circ} \mathbf{2 3 6}\right)$.

5 Soulignons l'importance de cette découverte: signe de troupeaux accompagnés d'un chien domestique. Outre la chasse d'animaux sauvages, les modes de subsistance des occupants du site de Doukanet el-Khoutifa reposent sur l'élevage des ovi-caprinés, cet ensemble procurant une grande quantité de ressources carnées. Le cheptel a été généralement abattu à l'âge adulte.

\section{Conclusion}

6 Les sites néolithiques de Redeyef (Table Sud), Kef el-Agab et Doukanet el-Khoutifa ont livré des restes humains qui ne sont pas tous en connexion anatomique, de l'industrie lithique et osseuse, des tessons de céramique et de la faune sauvage et domestique. En comparant les pourcentages de la faune sauvage et de la faune domestique dans les trois sites mentionnés, on constate la pratique d'un élevage intensif dans le site de Doukanet el-Khoutifa. Dans les deux autres sites, celui de Redeyef (Table Sud) et du Kef el-Agab, la faune sauvage est dominante. Les activités de chasse et de collecte (hélicidés terrestres, moules) ont été intensives dans ces deux derniers sites. Cependant, la production de produits animaliers à travers la domestication est assurée dans les trois sites mais avec des taux différent. Le chien n'est présent qu'à Doukanet el-Khoutifa. Un Bovini de petite taille (sans être pourtant Bos taurus) est présent dans le site de Redeyef (Table Sud) et à Doukanet el-Khoutifa. 


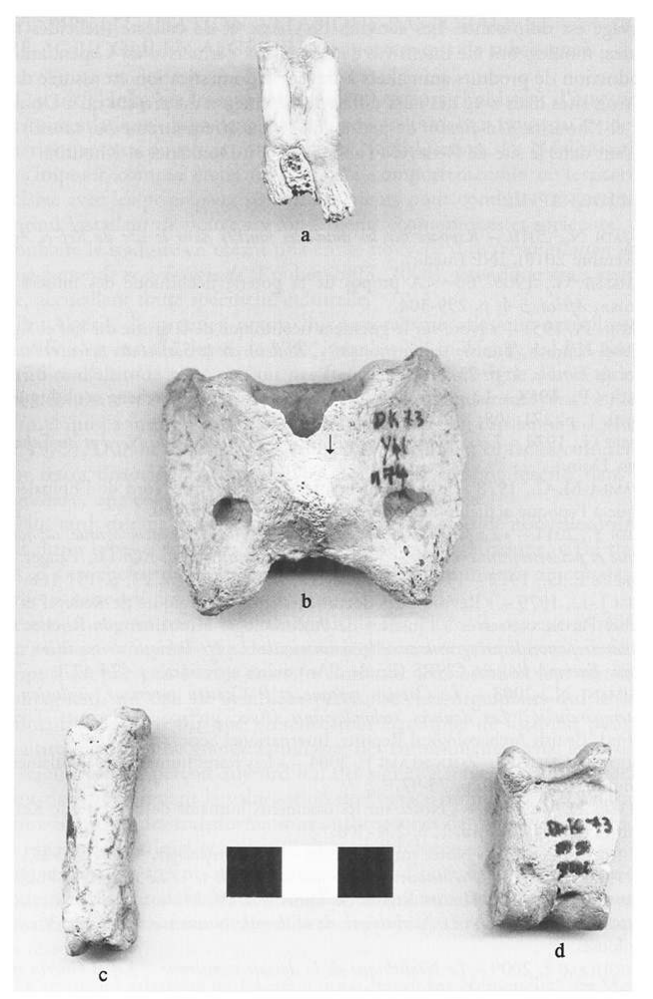

Fig. 5. PHOTO DE RESTES FAUNIQUES DU SITE DE DOUKANET EL-KHOUTIFA.

A : MOLAIRE (M1) INFÉRIEURE DE CAPRAL/OVIS.

B : ATLAS DE CAPRA HIRCUS AVEC DES TRACES DE DÉCOUPE,

C : PREMIÈRE PHALANgE D'AMMOTRAgUS LERVIA.

D : TALUS D'AMMOTRAgUS LERVIA.

\section{BIBLIOGRAPHIE}

AOUADI N., 2010 - Rapport sur les nouvelles fouilles dans le site de Kef el Agab (novembre 2010). INP (inédit).

AUMASSIP G., 1969-70 - «A propos de la poterie néolithique des musées de Tunis », Africa, 3-4, p. 299-304.

BARDIN P., 1951 - « Note sur le gisement néolithique de la grotte du Kef el Agab (Djebel Haïrech, Tunisie septentrionale) », Bulletin de la Société des Sciences Naturelles de Tunisie, 4, p. 23-24.

BARDIN P., 1953 - La grotte de Kef el Agab (Tunisie). Gisement néolithique », Libyca, 1, p. 271-308.

CAMPS G., 1974 - Les civilisations préhistoriques de l'Afrique du Nord et du Sahara, Paris, Doin.

CHAMLA M.-C., 1978 - « Le peuplement de l'Afrique du Nord de l'Épipaléolithique à l'époque actuelle », L'Anthropologie, 82/3, p. 385-430.

DRIDI Y., 2011 - «La faune holocène en Tunisie : Etudes archéozoologique, taphonomique et paléoenvironnementale », Résumé de communication RQM 6, Tanger. 
GOBERT E. G., 1912 - « L'abri de Redeyef », L'Anthropologie, 23, p. 151-168.

HEIM J.-L., 1979 - « Révision des documents anthropologiques de Redeyef et du Djebel Fartas, conservés à l'Institut de Paléontologie Humaine ", in Roubet C., Economie pastorale préagricole en Algérie orientale. Le Néolithique de tradition capsienne. Exemple l'Aurès, CNRS (Etude d'Antiquités africaines), p. 474-477.

RAHMANI N., 2003 - Le Capsien typique et le Capsien supérieur, évolution ou contemporanéité ? Les données technologiques (Avec préface de Jacques Tixier, Oxford, British Archaeological Reports, International Series : 1187.

RoUdesli-cHebbi S., zOUGHLAMI J., 2004 - « Les restes humains de Doukanet el Khoutifa », Africa, 20, p. 75-97.

VALLoIS H. V., 1953 - « Notes sur les ossements humains de la grotte de Kef El Agab (Tunisie) », Libyca, 1, p. 271-308.

VAUfREY R., 1933 - « Notes sur le Capsien », L’Anthropologie, 43, p. 457-483.

VAUfReY R., 1955 - Préhistoire de l'Afrique du Nord, t. 1, Le Maghreb, Publications de l'Institut des Hautes Etudes de Tunis, vol. IV, Masson, Paris.

zoughlami J., 1978 - Le Néolithique de la dorsale tunisienne. Thèse de $3^{\mathrm{e}}$ cycle, Toulouse.

zoUGHLAmi J. 2009 - Le Néolithique de la dorsale tunisienne - Kef el Guéria et sa région, Tunis, Centre de Publication Universitaire.

zOUGHLAMI J., CAMPS G., HARBI-RIAHI M., 1989 - Atlas Préhistorique de Tunisie, 4. Souk el Arba, Collection de l'Ecole Française de Rome : 81, Roma.

ZOUGHLAMI J., CHENORKIAN R., HARBI-RIAHI M., 1998 - Atlas Préhistorique de Tunisie, 11. Kairouan, Collection de l'Ecole Française de Rome : 81, Roma.

\section{INDEX}

Mots-clés : Néolithique, Tunisie 